\title{
Anabases
}

ANABASES Traditions et réceptions de l'Antiquité

28 | 2018

Varia

\section{Yannick SCOLAN, Le convive et le savant}

sophistes, rhéteurs, grammairiens et philosophes au banquet de Platon à Athénée

Mathilde Cambron-Goulet

\section{(2) OpenEdition}

Journals

Édition électronique

URL : http://journals.openedition.org/anabases/8282

DOI : $10.4000 /$ anabases.8282

ISSN : 2256-9421

Éditeur

E.R.A.S.M.E.

\section{Édition imprimée}

Date de publication : 9 novembre 2018

Pagination : 392-394

ISSN : 1774-4296

\section{Référence électronique}

Mathilde Cambron-Goulet, « Yannick scolan, Le convive et le savant: sophistes, rhéteurs, grammairiens et philosophes au banquet de Platon à Athénée », Anabases [En ligne], 28 | 2018, mis en ligne le 09 novembre 2018, consulté le 19 janvier 2021. URL : http://journals.openedition.org/anabases/8282 DOI : https://doi.org/10.4000/anabases.8282

Ce document a été généré automatiquement le 19 janvier 2021.

(c) Anabases 


\section{Yannick SCOLAN, Le convive et le savant}

\section{sophistes, rhéteurs, grammairiens et philosophes au banquet de Platon à Athénée}

Mathilde Cambron-Goulet

\section{RÉFÉRENCE}

Yannick SCOLAN, Le convive et le savant : sophistes, rhéteurs, grammairiens et philosophes au banquet de Platon à Athénée, Paris, Les Belles Lettres, 2017, 390 p., 55 euros / ISBN 9782251447025 .

1 Dans ce livre issu de sa thèse de doctorat, Scolan se questionne sur la manière dont la qualité de convive influence les discussions des savants mis en scène dans les banquets, et sur la manière dont le contexte convivial met à l'épreuve les savoirs et la sagesse des participants. Il dégage ainsi du $\sigma u \mu \pi o ́ \sigma i o v e t ~ d u ~ \delta \varepsilon \tilde{\pi} \pi v v$ une intention littéraire et des modalités d'écriture spécifiques : en dépit de la diversité des banquets, il est en effet possible selon Scolan de les caractériser par l'usage que leurs auteurs font de la table et du vin pour définir le savoir véritable, et par les divertissements qui les composent et qui constituent une sorte de propédeutique à la philosophie. Le banquet apparaît donc comme un genre littéraire codifié où la recherche commune de la vérité prime sur l'érudition, et où le convive idéal est celui qui désire savoir plutôt que celui qui sait, ce dernier étant incapable de prendre part à la recherche commune de la vérité, voire de respecter les usages symposiaques. 
2 Le chapitre 1 analyse la figure de Socrate au banquet, qui tant chez Platon que chez Xénophon permet de définir ce qu'est un philosophe, en opposant chez Platon ceux qui détiennent un savoir et ceux qui le cherchent, et en soulignant chez Xénophon l'impossible conversion à la vertu de celui qui ne sait pas adopter un comportement de symposiarque approprié (en l'occurrence Callias).

3 Les Propos de table de Plutarque et la pratique de la philosophie au banquet sont l'objet

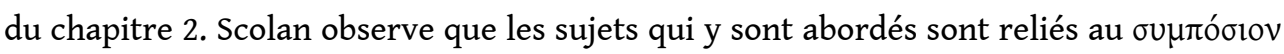
et $\mathrm{au}$ $\delta \varepsilon \tilde{\imath} \pi v 0 v$ , ou aux circonstances qui entourent la réunion. La plaisanterie, note-t-il, change le regard des convives sur leur environnement et suscite l'étonnement qui est à l'origine de la philosophie. Le recours à des arguments personnels constitue une pratique différente de la philosophie chez le savant et une propédeutique pour le profane. Pour Scolan, le banquet permet en effet de définir la vraie nature de la philosophie (chap. 3),

que le

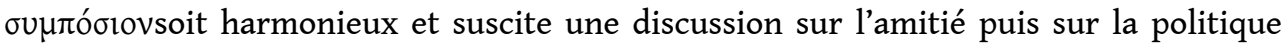
(Banquet des Sept Sages), ou soit tumultueux et rende la pratique de la philosophie impossible, puisque tourner le dos à l'amitié qui caractérise le banquet équivaut à se détourner de la philosophie (le Banquet ou les Lapithes).

4 L'érudition, qui semble parfois chez Athénée être mise en avant au détriment du banquet qui lui sert de cadre, s'harmonise avec les étapes du banquet, dont elle révèle la nature à la fois plaisante et agonistique, étudiée au chapitre 4. Le banquet apparaît ainsi comme une sorte de récréation intellectuelle, qui permet aux convives d'exercer leur sagacité en re-contextualisant des citations - une caractéristique que l'œuvre d'Athénée partage avec celles de Plutarque. En effet le banquet permet des raisonnements plus souples, raison pour laquelle celui-ci facilite la conversion à la philosophie : c'est l'objet du chapitre 5 . Scolan souligne la manière dont le mélange de sérieux et de rire favorise la pratique philosophique dans le Banquet de Platon et le Banquet de Xénophon. La plaisanterie permet d'introduire la parole philosophique au banquet, car comme il l'expose au chapitre 6, le convive idéal, dont Socrate constitue le

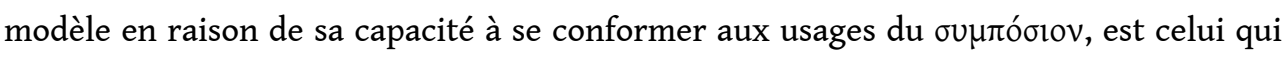
sait tirer profit des plaisirs du banquet et de juger adéquatement des plaisirs convenables. Aussi, pour Platon, Xénophon, Plutarque et Athénée, à condition de faire preuve de retenue, les plaisirs du banquet permettent-ils de s'exercer à la sagesse.

5 Le chapitre 7 porte quant à lui sur les imposteurs que le banquet permet de révéler, soit qu'ils se disqualifient comme philosophes en contrevenant aux codes du banquet, soit que leur refus de participer à la recherche commun de la vérité montre du même coup leur refus du rôle de convives : ainsi les rhéteurs et les grammairiens, dont l'érudition est fort éloignée de la commune recherche de la vérité, dans les Propos de table; les philosophes cyniques, dont le franc-parler met à mal l'harmonie du groupe et dont la voracité trahit la fausse ascèse, dans les Déipnosophistes, et tant les philosophes incapables de mettre en pratique leurs idéaux que les grammairiens dont l'érudition rend les interventions incohérentes, chez Lucien.

6 L'étude des modèles littéraires de Plutarque et Athénée (chap.8), remettant en question l'autorité de Platon, amène Scolan à souligner le recours au modèle homérique tant chez Athénée que chez Plutarque. Les deux auteurs traitent toutefois Homère de manière différente : Plutarque le cite afin de connaître les usages symposiaques et de soutenir la discussion, tandis qu'Athénée y voit un modèle littéraire permettant de 
définir le banquet par l'intention pédagogique sous-jacente et par la mise en scène d'une assemblée harmonieuse de personnages de condition et d'âge variés, visant la sagesse de manière plaisante - même si Scolan note que les Déipnosophistes s'appuient davantage sur les Propos de table de Plutarque, auxquels Athénée surimpose la culture impériale des arts libéraux.

7 L'ouvrage est d'excellente facture (vaste bibliographie classifiée, index détaillés);

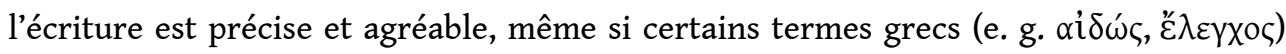
ne sont pas commentés. Avec ce livre, Scolan met en lumière l'intention programmatique qui caractérise le banquet comme genre littéraire, et qui consiste à «tirer du contexte symposiaque lui-même les sujets qui [permettent] d'exercer avec souplesse et bonne humeur son esprit à la philosophie [et conduisent] à la définition du savoir véritable » (p.324).

\section{AUTEUR}

\section{MATHILDE CAMBRON-GOULET}

Université du Québec à Montréal

cambron-goulet.mathilde@uqam.ca 